\title{
Results of electrophysiologic testing and long-term prognosis in patients with coronary artery disease and aborted sudden death
}

The purpose of this study was to evaluate the results of electrophysiologic testing and the long-term prognosis of 56 patients with coronary artery disease who presented with aborted sudden death unrelated to acute myocardial infarction. The mean age of the patients was $62 \pm 8$ years ( \pm standard deviation) and 48 were men. The mean left ventricular ejection fraction was $0.34 \pm 0.16$. During the baseline electrophysiology test, sustained monomorphlc ventricular tachycardia (VT) was inducible in 22 patients who then underwent electropharmacologic testing: 11 patients were treated with antiarrhythmic drugs that suppressed the induction of VT or resulted in the VT becoming hemodynamically stable; 10 patients who falled drug testing recelved an automatic Implantable cardloverter/deflbrillator (AICD); one patient underwent endocardial resection. Among 34 patients who did not have inducible sustained VT, a precipitant of cardlac arrest (severe ischemia, proarrhythmia) was identlfied and was corrected in 9 of 34 . An AICD was recommended in the remaining 25 patients; however, nine patients refused and were treated empirically with antiarrhythmic drugs. The mean follow-up was $22 \pm 12$ months. The 2-year actuarial incidence of sudden death was $31 \%$ in patients who were treated with drugs based on the results of electropharmacologic testing, $26 \%$ in patients who were treated with antiarrhythmic drugs on an empiric basis, $0 \%$ among patients in whom a correctable etiology for the cardiac arrest was identified, and $9 \%$ among patients who underwent implantation of an AICD. The 3-year actuarial incidence of sudden death among the 20 patients treated with antiarrhythmic drugs was $53 \%$, compared with $9 \%$ among the 26 patients who underwent AICD implantation $(p=0.03)$. In conclusion, antiarrhythmic therapy, whether guided by electrophysiologic testing or administered on an empiric basis, is associated with a high incidence of recurrent sudden death in patients with coronary artery disease and aborted sudden death. Implantation of an AICD may be advisable in all patients with coronary artery disease and aborted sudden death in whom a correctable precipitant cannot be identified. (AM HEART J 1991;122: 1001.)

João Sousa, MD, Shimon Rosenheck, MD, Hugh Calkins, MD, Michael de Buitleir, MD, Stephen Schmaltz, MPH, Alan Kadish, MD, and Fred Morady, MD. Ann Arbor, Mich.

Although several previous studies have described the results of electrophysiologic testing and the prognosis in patients with aborted sudden death, many of these studies ${ }^{1-5}$ have included heterogeneous patient populations with various types of heart disease and the majority were performed before the widespread availability of the automatic implantable cardiovert-

From the Division of Cardiology and Clinical Research Center, Department of Internal Medicine, University of Michigan Medical Center.

Supported in part by Grant 5M01-RR00042-25 from the National Institutes of Health, Bethesda, Md.

Received for publication Feb. 26, 1991; accepted April 8, 1991.

Reprint requests: Fred Morady, MD, University of Michigan Medical Center, 1500 East Medical Center Drive, B1 F245, Ann Arbor, MI 48109-0022. 4/1/31115 er/defibrillator (AICD). Several issues relevant specifically to patients with coronary artery disease remain to be clarified.

The purpose of this study was to address the following questions in patients with coronary artery disease and a recent history of aborted sudden death: (1) How often is an adequate response to electropharmacologic testing obtained in patients with inducible, sustained monomorphic ventricular tachycardia (VT)? (2) How often can a potentially reversible etiology for the cardiac arrest be identified in patients without inducible VT? (3) How does longterm prognosis relate to the inducibility of VT, to a response to drug-testing, to treatment with coronary revascularization in patients without inducible VT, and to the use of the AICD? 
Table I. Comparison of patients with and without inducible sustained monomorphic ventricular tachycardia

\begin{tabular}{lccc}
\hline \multicolumn{1}{c}{$\begin{array}{c}\text { Clinical } \\
\text { characteristics }\end{array}$} & $\begin{array}{c}\text { SMVT } \\
(n=22)\end{array}$ & $\begin{array}{c}\text { Without } \\
(n=34)\end{array}$ & $p$ Value \\
\hline Age $(\mathrm{yr})$ & $62 \pm 8^{*}$ & $62 \pm 8$ & $\mathrm{NS}$ \\
Sex (m/f) & $20 / 2$ & $28 / 6$ & $\mathrm{NS}$ \\
Previous infarction & $19(86 \%)$ & $26(76 \%)$ & $\mathrm{NS}$ \\
Previous revascularization & 5 & 6 & $\mathrm{NS}$ \\
Aneurysm & $15(68 \%)$ & $11(32 \%)$ & $<0.01$ \\
CHF & $19(86 \%)$ & $18(53 \%)$ & $<0.01$ \\
LVEF & $0.30 \pm 0.15$ & $0.38 \pm 0.16$ & $\mathrm{NS}$ \\
No. of obstructed CAs & $2.5 \pm 0.75$ & $2.0 \pm 0.8$ & $<0.03$ \\
\hline
\end{tabular}

CA, Coronary artery; CHF, congestive heart failure; LVEF, left ventricular ejection fraction; SMVT, sustained monomorphic ventricular tachycardia.

*Mean \pm standard deviation.

\section{METHODS}

Patient population. The subjects of this study were 56 consecutive patients with coronary artery disease who were referred for evaluation of out-of-hospital aborted sudden death unrelated to acute myocardial infarction between September 1986 and September 1989. The mean age of these patients was $62 \pm 8$ years ( \pm standard deviation), and there were 48 men and 8 women. Each patient required electrical cardioversion, cardiopulmonary resuscitation, and/or direct-current countershock during the cardiac arrest. Patients with electrocardiographic evidence of a new transmural myocardial infarction were excluded from the study. A history of previous myocardial infarction was present in 45 patients $(80 \%)$. Eleven patients had undergone previous revascularization procedures (nine with hypass surgery and two with angioplasty). Ten patients were taking antiarrhythmic drugs at the time of the cardiac arrest, including quinidine (four), procainamide (two), disopyramide, tocainide, mexiletine, or amiodarone. Signs and symptoms of congestive heart failure were present in 37 patients. Fifty-three patients had angiographicallyproven coronary artery disease. In three patients, each of whom had a history of myocardial infarction, coronary angiography was not performed. There were 26 patients with three-vessel disease, 15 with two-vessel disease, and 13 with one-vessel disease. The mean left ventricular ejection fraction as determined by a contrast ventriculogram or a radionuclide angiogram was $0.34 \pm 0.16$. Angiographic evidence of a left ventricular aneurysm was present in 26 pa tients.

Electrophysiologic testing. Electrophysiology tests were performed in the fasting, unsedated state after informed consent had been obtained from each patient. The electrophysiology tests were performed at a mean of $42 \pm 76$ days after the cardiac arrest. All antiarrhythmic drugs were discontinued at least 5 half-lives before the test. Three quadripolar electrode catheters were inserted into a femoral vein and were positioned in the right atrium, His bundle, and right ventricle. The surface electrocardiographic leads and intracardiac electrograms were recorded on a Siemens Elema Mingograph 7 recorder (Siemens Elema AB, Solna, Sweden). A programmable stimulator (Bloom Associates, Ltd., Reading, Pa.) was used to deliver rectangular pulses $2 \mathrm{msec}$ in duration at twice the diastolic threshold. Programmed ventricular stimulation was performed at the right ventricular apex and at the outflow tract or septum using one, two, and three extrastimuli de livered after an eight-beat drive train at cycle lengths of 400 and $600 \mathrm{msec}$. For each extrastimulus, diastole was scanned in $10 \mathrm{msec}$ decrements to the point of ventricular refractoriness or until a coupling interval of $200 \mathrm{msec}$ was attained. If monomorphic VT was not inducible, programmed stimulation was repeated during an infusion of isoproterenol at a rate of 1 to $4 \mu \mathrm{g} / \mathrm{min}$ titrated to maintain a heart rate of 110 to 120 beats $/ \mathrm{min}$.

Sustained VT was defined as VT with a duration $>30$ seconds or requiring termination because of hemodynamic collapse. Nonsustained VT was defined as VT having a duration of 6 beats to 30 seconds. Induced VT was considered monomorphic if the QRS morphology was constant and the cycle length was $>200$ msec. Polymorphic VT was defined as VT that had a variable QRS morphology. Ventricular fibrillation was defined as an arrhythmia having a disorganized QRS morphology and a cycle length $<200$ msec.

Patient management. All patients who had inducible, sustained monomorphic V'T underwent serial electropharmacologic testing. Drugs were tested based on each patient's history and previous clinical response. A Class IA or IC drug was usually used first, followed by amiodarone, then by a combination of amiodarone and a Class I drug. The tests were performed after oral loading adequate to achieve a steady-state drug level. Patients treated with amiodarone were retested after 1 to 2 weeks of loading with 1.2 to $2.4 \mathrm{gm} /$ day. The dosages of the other drugs were: quinidine, 1.2 to $1.6 \mathrm{gm} /$ day; mexiletine, 600 to $900 \mathrm{mg} /$ day; procainamide, 2 to $3 \mathrm{gm} /$ day; encainide, $105 \mathrm{mg} /$ day. The response to a drug was considered adequate if sustained VT was no longer inducible or, in the case of amiodarone, if only relatively slow and hemodynamically stable VT (mean blood pressure $>70 \mathrm{~mm} \mathrm{Hg}$ ) was inducible. ${ }^{6}$ In patients who failed electropharmacologic testing, AICD implantation was recommended.

Electropharmacologic testing was not performed in patients who had inducible polymorphic VT or ventricular fibrillation (VF), because these arrhythmias are often a nonspecific response to programmed stimulation and their clinical significance is unclear. ${ }^{7,8}$

In patients in whom monomorphic VT could not be induced, reversible causes for the cardiac arrest were sought. These included proarrhythmia (assumed when the episode of cardiac arrest occurred after the initiation of the drug or after an increase in dosage of an antiarrhythmic drug known to cause serious proarrhythmia) or cardiac ischemia (assumed when the cardiac arrest was preceded by angina, if coronary angiography demonstrated critical coronary lesions, and if functional studies revealed evidence of is chemia). In these patients, management included discontinuation of the proarrhythmic drug or myocardial revas- 


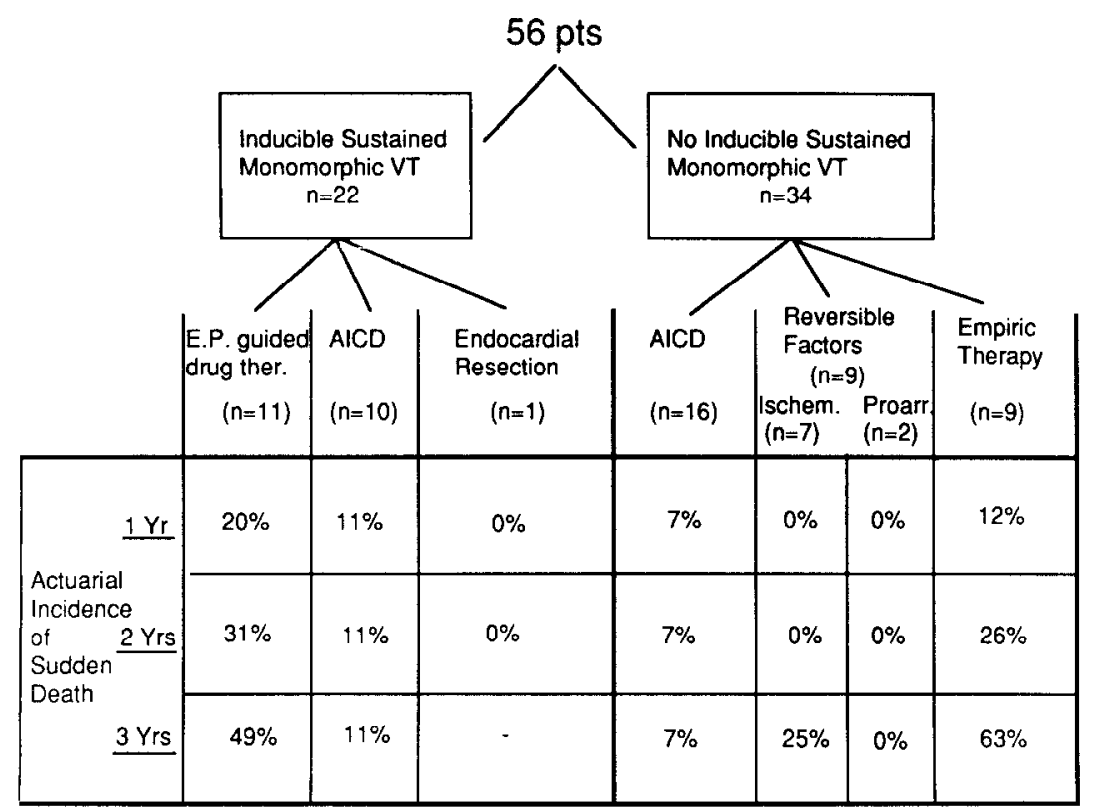

Fig. 1. Management and outcome of patients. Actuarial incidence of sudden death, according to treatment. E.P., Electrophysiologic; $A I C D$, automatic implantable cardioverter/defibrillator; Ischem., ischemia; Proarr., proarrhythmia; VT, ventricular tachycardia.

cularization without implantation of an AICD if the left ventricular ejection fraction was $>0.35$. In patients who had neither inducible VT nor an identifiable reversible cause, implantation of an AICD was recommended. If AICD implantation was declined, empiric antiarrhythmic therapy was initiated.

All patients were followed on a regular basis by one of the investigators or by their referring physicians. To obtain follow-up information for this study, patients were interviewed in person or by telephone. Sudden death was defined as unexpected natural death occurring during sleep or within 1 hour of the patient's collapse. In patients who had an AICD, the number of shocks was noted.

Statistical analysis. Continuous data are expressed as the mean \pm 1 standard deviation. Comparisons were performed using chi square analysis or Student's $t$ test. Actuarial survival curves were constructed by the Kaplan-Meier method and were analyzed with the log-rank test. If death was not sudden or there was a change in therapy, the patient was censored. Predictors of inducible monomorphic VT and recurrent sudden death were evaluated with the Cox regression model. ${ }^{9}$ A $p$ value $<0.05$ was considered significant.

\section{RESULTS}

Electrophysiologic testing. Sustained monomorphic VT was induced by ventricular programmed stimulation in 22 of 56 patients $(40 \%)$. The VT was induced by two extrastimuli in nine patients (41\%), by three extrastimuli in 11 patients $(50 \%)$, and by two extrastimuli during isoproterenol infusion in two patients $(9 \%)$. The VT cycle length varied between
210 and $420 \mathrm{msec}$ (mean $273 \pm 52 \mathrm{msec}$ ). The induced VT had a right bundle branch block morphology in 12 patients and a left bundle branch block morphology in 10. Among the 34 patients in whom sustained monomorphic VT could not be induced, sustained polymorphic VT was induced in two patients, ventricular fibrillation was induced in four, nonsustained VT was induced in 10 patients (polymorphic in nine and monomorphic in one), and no ventricular arrhythmias could be induced in 18 patients.

The patients who did and did not have inducible sustained, monomorphic VT are compared in Table I. Three clinical variables were associated with the presence of inducible monomorphic VT: evidence of a left ventricular aneurysm $(p<0.01)$, the number of diseased coronary arteries ( $p<0.03$ ), and evidence of heart failure $(p<0.01)$. Other clinical variables including age, sex, history of previous myocardial infarction, left ventricular ejection fraction, previous revascularization procedures, and prior antiarrhythmic drug therapy were similar in the two groups.

Drug testing and management (Fig. 1). All patients in whom sustained monomorphic VT could be induced underwent serial electropharmacologic testing with a mean of $1.8 \pm 0.8$ drugs. Eleven patients (50\%) had an adequate response: two with quinidine, four with amiodarone, and five with a combination of amiodarone and a Class I drug. The patients who failed electropharmacologic testing underwent im- 


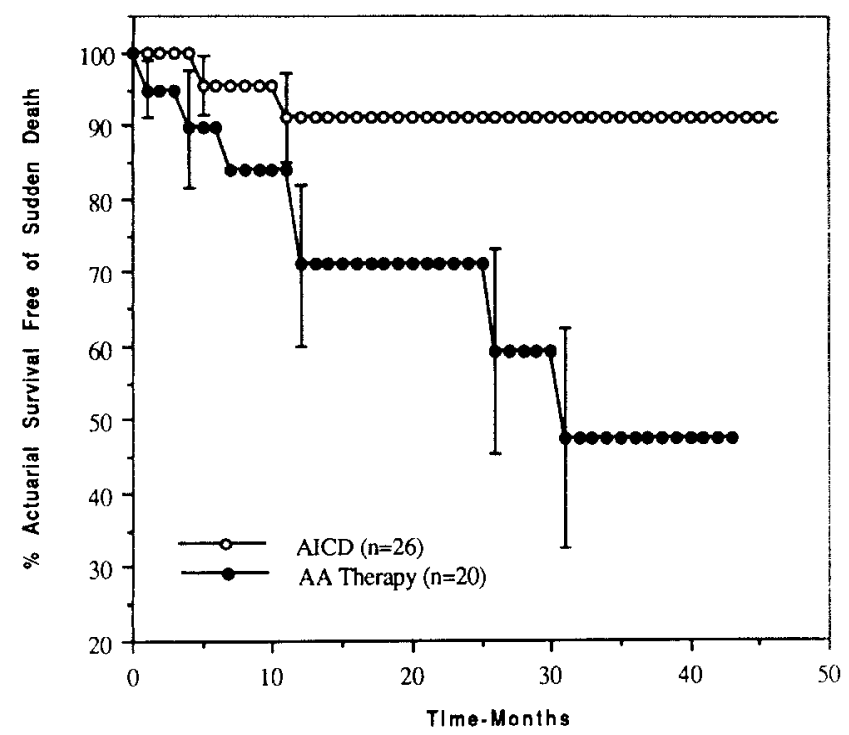

Fig. 2. Kaplan-Meier actuarial survival curves of patients who had an $\operatorname{AICD}(n=26)$ and of patients who had antiarrhythmic drug therapy $(n=20)$. There was a significant difference at 3 years of follow-up $(p=0.03)$ between the two groups. Patients were censored if death was not sudden.

plantation of an AICD (10 patients) or had endocardial resection (one patient). Four of these patients also underwent bypass surgery. There were no significant differences in clinical or electrophysiologic characteristics between the patients who had an adequate response to electropharmacologic testing and those who did not.

Among the 34 patients without inducible sustained monomorphic VT, a correctable factor that was likely to be responsible for the cardiac arrest was identified in nine patients. Proarrhythmia was presumed to be responsible for their cardiac arrest in two patients who had been treated with quinidine and who had a prolonged QT interval. In seven patients whose mean left ventricular ejection fraction was $0.50 \pm 0.13$ (range 0.35 to 0.69 ), ischemia was identified as the probable precipitant of cardiac arrest; revascularization was performed with bypass surgery in one patient and by percutaneous coronary angioplasty in six. These patients were also treated on a long-term basis with $\beta$-adrenergic blocking or calcium antagonist agents. Sixteen other patients without inducible, sustained monomorphic VT underwent implantation of an AICD (with concomitant bypass surgery in six). Nine patients declined AICD implantation and were treated empirically with amiodarone, quinidine, encainide, or procainamide.

Long-term follow-up (Fig. 1). The mean duration of follow-up was $22 \pm 12$ months. There were a total of
15 deaths, with 10 being sudden. The overall actuarial rate of sudden death was $14 \%$ at 1 and 2 years, and $30 \%$ at 3 years. The actuarial rates of sudden death in the various subgroups of patients are shown in Fig. 1. The incidence of sudden death was not significantly different among these subgroups, except for the patients who had a reversible cause of cardiac arrest, and for those who had a significantly lower incidence of sudden death at 2 years of follow-up. None of the patients had other significant arrhythmic events, such as sustained VT not associated with loss of consciousness.

A total of 26 patients underwent AICD implantation: 10 with inducible sustained, monomorphic VT who failed electropharmacologic testing and 16 without inducible VT in whom there was no apparent reversible factor for the cardiac arrest. The 3-year incidence of sudden death was $9 \%$ in these 26 patients, compared with $53 \%$ in the 20 patients who received antiarrhythmic therapy $(p=0.03)$ (Fig. 2). Among the patients who received an AICD, in those who had inducible sustained, monomorphic VT, eight received shocks $(80 \%)$, while in those without inducible VT, seven received shocks ( $44 \%$ ). Symptoms of cerebral hypoperfusion preceded $80 \%$ of the shocks. Statistical analysis failed to show any variable that had independent predictive value for sudden death in the first 3 years of follow-up among the clinical, angiographic, electrophysiologic, and therapeutic variables considered.

\section{DISCUSSION}

Patients with inducible VT. The results of this study demonstrate that sustained, monomorphic VT can be induced in slightly less than $50 \%$ of patients with coronary artery disease following an episode of aborted sudden death. The ability to induce sustained, monomorphic VT correlates with the presence of a left ventricular aneurysm, congestive heart failure, and triple-vessel disease. Based on electropharmacologic testing, an "effective" antiarrhythmic drug was identified in $50 \%$ of these patients. However, the results of long-term therapy guided by electropharmacologic testing were disappointing, with approximately a $50 \%$ incidence of sudden death at 3 years of follow-up. Patients who failed electropharmacologic testing received an AICD, and although the 3-year incidence of sudden death in these patients was $11 \%$, the majority experienced at least one AICD discharge during follow-up. This suggests that the incidence of sudden death in these patients may have been considerably higher had an AICD not been implanted.

Patients without inducible VT. Among the $60 \%$ of 
patients in this study who did not have inducible, sustained, monomorphic VT, a potentially correctable cause of cardiac arrest, namely ischemia or proarrhythmia, could be identified in one fourth. These patients, all of whom had left ventricular ejection fractions of $\geq 0.35$, had an excellent prognosis after revascularization or discontinuation of treatment with the proarrhythmic agent, and therefore it appears that specific antiarrhythmic therapy may not be necessary if a reversible cause of cardiac arrest can be identified and corrected and if the patient does not have severe left ventricular dysfunction.

In the patients who had neither inducible VT nor an identifiable precipitant of cardiac arrest, the factors that triggered the episode of aborted sudden death remain conjectural. Empiric antiarrhythmic therapy in these patients was associated with a $63 \%$ incidence of sudden death at 3 years of follow-up, compared with a 7\% 3-year incidence of sudden death in comparable patients who received an AICD. Furthermore, approximately half of the patients who had an AICD experienced one or more AICD discharges during follow-up, suggesting that the incidence of sudden death may have been higher than $7 \%$ if an AICD had not been implanted.

Incidence of inducible vT. The incidence of inducible VT in patients who have survived an episode of out-of-hospital cardiac arrest has been reported to vary between $55 \%$ and $81 \%$, depending on the patient population, the programmed stimulation protocol, and the end points of programmed stimulation. ${ }^{1-5,10-13}$ The present study differed from these prior studies in two ways. First, the subjects of the present study were a homogeneous group of patients with chronic coronary artery disease and out-of-hospital cardiac arrest. Second, because the inducibility of nonsustained or polymorphic VT/VF is of unclear significance and because these arrhythmias may often represent a nonspecific response to programmed stimulation, ${ }^{7,8}$ only sustained, monomorphic VT was considered to be a positive finding during electrophysiologic testing. These differences may explain why the incidence of inducible VT in the present study $(40 \%)$ was lower than the incidence reported in several prior studies.

Unreliability of electropharmacologic testing. In prior studies, patients with sustained VT who were treated long-term with antiarrhythmic drugs that suppressed the induction of VT were found to have a relatively good prognosis. ${ }^{2-4,} 6,14$ However, in the present study, there was a $49 \%$ 3-year incidence of sudden death in the group of patients whose drug therapy was guided by electropharmacologic testing. This suggests that therapy based on electropharmacologic testing may not be as reliable long-term in patients with coronary artery disease who have had aborted sudden death as in patients who present with sustained VT. Differences in left ventricular function, the susceptibility to ischemic events, and changes in the myocardial arrhythmia substrate over time may be among the factors that could account for the unreliability of therapy based on electropharmacologic testing in the patients in this study.

Reversible causes of cardiac arrest. The inability to induce sustained, monomorphic VT in a patient with coronary artery disease who has survived a cardiac arrest may suggest that the anatomic substrate for sustained VT is not present and that the cardiac arrest was precipitated by a reversible abnormality such as ischemia, an electrolyte disturbance, or drug proarrhythmia. However, among the patients in this study in whom VT could not be induced, evidence suggestive of a reversible precipitant of cardiac arrest was found in only $25 \%$. As has been reported previously, ${ }^{11}$ the prognosis of these patients was good with therapy directed only at correcting the abnormality presumed to have triggered the cardiac arrest, i.e., ischemia or proarrhythmia.

The mechanism of cardiac arrest is unclear in the patients who had neither inducible VT nor evidence of a precipitant abnormality. Nevertheless, it is clear that these patients remain at risk of sudden death and that empiric antiarrhythmic drug therapy is inappropriate in these patients, since the incidence of sudden death was $63 \%$ at 3 years of follow-up when patients were treated in this fashion. This high incidence of sudden death suggests that AICD implantation may be the optimal therapy in these patients.

Prognosis after AICD implantation. Among the 26 patients who underwent implantation of an AICD, the incidence of sudden death at 2 years of follow-up was $9 \%$. In contrast, previous studies have reported that the 2-year incidence of sudden death in patients who have received an AICD is $<5 \% .{ }^{15-19}$ This difference in survival following AICD implantation may be attributable to differences in the patient population of various studies. Unlike most prior studies, the present study included only patients who had already had an episode of out-of-hospital cardiac arrest and therefore were at high risk of having a recurrence.

Despite the significant incidence of sudden death following AICD implantation, the fact that a majority of patients experienced at least one discharge from the AICD during follow-up suggests that the AICD was probably effective in reducing the incidence of sudden death. However, it must be acknowledged that some AICD discharges may not have been triggered by life-threatening VT/VF, especially 
among the $20 \%$ of patients who received a shock that was not preceded by any symptoms of cerebral hypoperfusion. A comparison of the incidence of sudden death in patients who were treated with an antiarrhythmic drug and in those who received an AICD (53\% versus $9 \%$ at 3 years of follow-up) indicates that the AICD may be the treatment of choice in patients similar to the ones in this study.

Predictive value of the left ventricular ejection fraction. In accord with their history of cardiac arrest, the majority of patients in this study had left ventricular dysfunction. Whereas prior studies have demonstrated that left ventricular ejection fraction is a significant predictor of sudden death, ${ }^{3,4}$ this was not the case in the present study. However, the absence of any correlation between ejection fraction and sudden death in the patients in this study may be explained by the fact that all of these patients had already had an aborted episode of sudden death before entry into the study. Therefore they had already demonstrated themselves to be the high-risk patients that other studies would have predicted to be at risk.

Conclusions. Patients who have coronary artery disease and who have survived an out-of-hospital cardiac arrest remain at high risk of sudden death whether or not sustained monomorphic VT can be induced during a baseline electrophysiology test, except for the relatively small subgroup of patients in whom there is evidence of a correctable precipitant such as ischemia or proarrhythmia. Because antiarrhythmic drug therapy, whether guided by electropharmacologic testing or administered on a empiric basis, is associated with a high incidence of sudden death, AICD implantation may be advisable in all patients with coronary artery disease and aborted sudden death in whom a correctable precipitant cannot be identified.

The authors thank Marion Maguire for expert assistance in the preparation of the manuscript.

\section{REFERENCES}

1. Morady F, Scheinman M, Hess D, Sung R, Shen E, Shapiro W Electrophysiology testing in the management of survivors of out-of-hospital cardiac arrest. Am J Cardiol 1983;51:85-9.

2. Ruskin J, DiMarco JP, Garan H. Out-of-hospital cardiac arrest. Electrophysiologic observations and selection of long term antiarrhythmic therapy. N Engl J Med 1980;303:607-13

3. Wilber D, Garan H, Finkelstein D, Kelly E, Newell J, McGov ern B, Ruskin J. Out-of-hospital cardiac arrest. Use of electrophysiologic testing in the prediction of long-term outcome. N Engl J Med 1988;318:19-24.
4. Furukawa T, Rozanski J, Nogami A, Moroe K, Giosselin A. Lister J. Time-dependent risk of and predictors for cardiac arrest recurrence of survivors of out-of-hospital cardiac arrest with chronic coronary artery disease. Circulation 1989;80:599. 608 .

5. Roy D, Waxman H, Kienzle M, Buxton A, Marchlinski F, Josephson $\mathrm{M}$. Clinical characteristics and long-term follow-up in 119 survivors of cardiac arrest: relation to inducibility at elec trophysiologic testing. An J Cardiol 1983;52:969-74.

6. Waller TJ, Kay HR, Spielman SR, Kutalek SP, Greenspan AM, Horowitz LN. Reduction in sudden death and total mortality by antiarrhythmic therapy evaluated by electrophysiologic drug testing: criteria of efficacy in patients with sustained ventricular tachyarrhythmia. J Am Coll Cardiol 1987;10:83-9.

7. Wellens H, Brugada P. Stevenson W. Programmed electrical stimulation of the heart in patients with life-threatening ven tricular arrhythmias: what is the significance of induced arrhythmias and what is the correct stimulation protocol? Circulation 1985;72:1-7.

8. DiCarlo L, Morady F, Schwartz A, Shen E, Baerman J, Krol $R$, Scheinman M, Sung R. Clinical significance of ventricular fibrillation/flutter induced by ventricular programmed stim ulation. AM HEART J 1985;109:1-8.

9. Cox DR, Oakes D. Analysis of survival data. London: Chap man and Hall, 1984: chapters 4 and 7.

10. Goldstein S, Landis J, Leighton R, Ritter G, Vasu C, Lantis A Serokman $R$. Characteristics of the resuscitated out-of-hospi tal cardiac arrest victim with coronary heart discase. Circula tion 1981;64:977-84

11. Morady F, DiCarlo L, Winston S, Davis J, Scheinman M. Clinical features and prognosis of patients with out-of-hospital cardiac arrest and a normal electrophysiology study. J Am Coll Cardiol 1984;4:39-44.

12. Freedman R, Swerdlow C, Soderholm-Difatte V, Mason J. Prognostic significance of arrhythmia inducibility or nonin ducibility at initial electrophysiologic study in survivors of cardiac arrest. Am J Cardiol 1988;61:578-82.

13. Poole J, Mathisen T, Kudenchuk P, McNulty J, Swerdlow C Bardy $\mathrm{G}$, Greene $\mathrm{H}$. Long term outcome in patients who sur vive out-of -hospital ventricular fibrillation and undergo electrophysiologic studies: evaluation by electrophysiologic subgroups. J Am Coll Cardiol 1990;16:657-65.

14. Horowitz L, Greenspan A, Spielman S, Webb C, Morganroth J, Rotmensch H, Sokoloff N, Rae A, Segal B, Kay H. Usefulness of electrophysiologic testing in evaluation of amiodarone therapy for sustained ventricular tachyarrhythmias associated with coronary heart disease. Am J Cardiol 1985;55:367. 71.

15. Lehmann M, Steinman R, Schuger C, Jackson K. The automatic implantable cardioverter defibrillator as antiarrhythmic treatment modality of choice for survivors of cardiac arrest unrelated to acute myocardial infarction. Am J Cardiol 1988;62:803-5

16. Mirowski $M$. The automatic implantable cardioverter-defibrillator: an overview. J Am Coll Cardiol 1985;6:461-6.

17. Annual post-FDA approval report on the automatic implantable cardioverter defibrillator (AICD) system. St. Paul, Minn. Cardiac Pacemakers, Inc., 1988.

18. Kelly PA, Cannon DS, Garan $\mathrm{H}$, et al. The automatic im plantable cardioverter-defibrillator: efficacy, complications, and survival in patients with malignant ventricular arrhyth mias. J Am Coll Cardiol 1988;11:1278-86.

19. Winkle R, Mead H, Duder M, et al. Long-term outcome with the automatic implantable cardioverter-defibrillator. J Am Coll Cardiol 1989;13:1356-61. 